\title{
Dupilumab for the Treatment of Lichen Planus
}

\author{
Bobak T. Pousti, MD, MBA; Annie Jin, MD; Lindsay Sklovar, MD; Kevin T. Savage, MD; Lisa L. Zhai, BS; \\ Nicholas K. Mollanazar, MD, MBA; Candrice R. Heath, MD
}

\section{PRACTICE POINTS}

- Lichen planus (LP) is an inflammatory mucocutaneous disorder that can present across various regions of the body with pruritic, purple, polygonal papules or plaques.

- The proposed pathogenesis of LP involves autoimmune destruction of epidermal basal keratinocytes.

- The immunomodulatory properties of dupilumab have been shown to aid various inflammatory cutaneous conditions.

To the Editor:

Lichen planus (LP) is an inflammatory mucocutaneous disorder that primarily affects adults aged 30 to 60 years. ${ }^{1}$ It can present across various regions such as the skin, scalp, oral cavity, genitalia, nails, and hair. It classically presents with pruritic, purple, polygonal papules or plaques. The proposed pathogenesis of this condition involves autoimmune destruction of epidermal basal keratinocytes. ${ }^{2}$ Management involves a stepwise approach, beginning with topical therapies such as corticosteroids and phototherapy and proceeding to systemic therapy including oral corticosteroids and retinoids. Additional medications with reported positive results include immunomodulators such as cyclosporine, tacrolimus, and mycophenolate mofetil. ${ }^{2-4}$ Dupilumab is a biologic immunomodulator and antagonist to the IL- $4 R \alpha$ on helper $\mathrm{T}$ cells $\left(\mathrm{T}_{\mathrm{H}} 1\right)$. Although indicated for the treatment of moderate to severe atopic dermatitis, this medication's immunomodulatory properties have been shown to aid various inflammatory cutaneous conditions, including prurigo nodularis. ${ }^{5-9}$ We present a case of dupilumab therapy for treatment-refractory LP.

A 52-year-old man presented with a new-onset progressive rash over the prior 6 months. He reported no history of atopic dermatitis. The patient described the rash as "severely pruritic" with a numeric rating scale itch intensity of $9 / 10$ ( 0 being no itch; 10 being the worst itch imaginable). Physical examination revealed purple polygonal scaly papules on the arms, hands, legs, feet, chest, and back (Figure 1).

Three biopsies were taken, all indicative of lichenoid dermatitis consistent with LP. Rapid plasma reagin as well as HIV and hepatitis C virus serology tests were negative. Halobetasol ointment, tacrolimus ointment, and oral prednisone (28-day taper starting at $40 \mathrm{mg}$ ) all failed. Acitretin subsequently was initiated and failed to provide any benefit. The patient was unable to come to clinic 3 times a week for phototherapy due to his work schedule.

Drs. Pousti, Jin, Sklovar, Heath, and Ms. Zhai are from the Department of Dermatology, Lewis Katz School of Medicine at Temple University, Philadelphia, Pennsylvania. Dr. Savage is from Drexel University College of Medicine, Philadelphia. Dr. Mollanazar is from the Department of Dermatology, Perelman School of Medicine, University of Pennsylvania, Philadelphia.

Drs. Pousti, Jin, Sklovar, Savage, and Ms. Zhai report no conflicts of interest. Dr. Mollanazar reports serving as an investigator in trials sponsored by Regeneron Pharmaceuticals and Sanofi. Dr. Heath reports serving as a consultant on the advisory board for Cassiopea and Pfizer.

Correspondence: Bobak T. Pousti, MD, MBA, Department of Dermatology, Lewis Katz School of Medicine at Temple University, 1316 W Ontario St, 1st Floor, Philadelphia, PA 19140 (bobak.pousti@temple.edu).

doi:10.12788/cutis.0232 

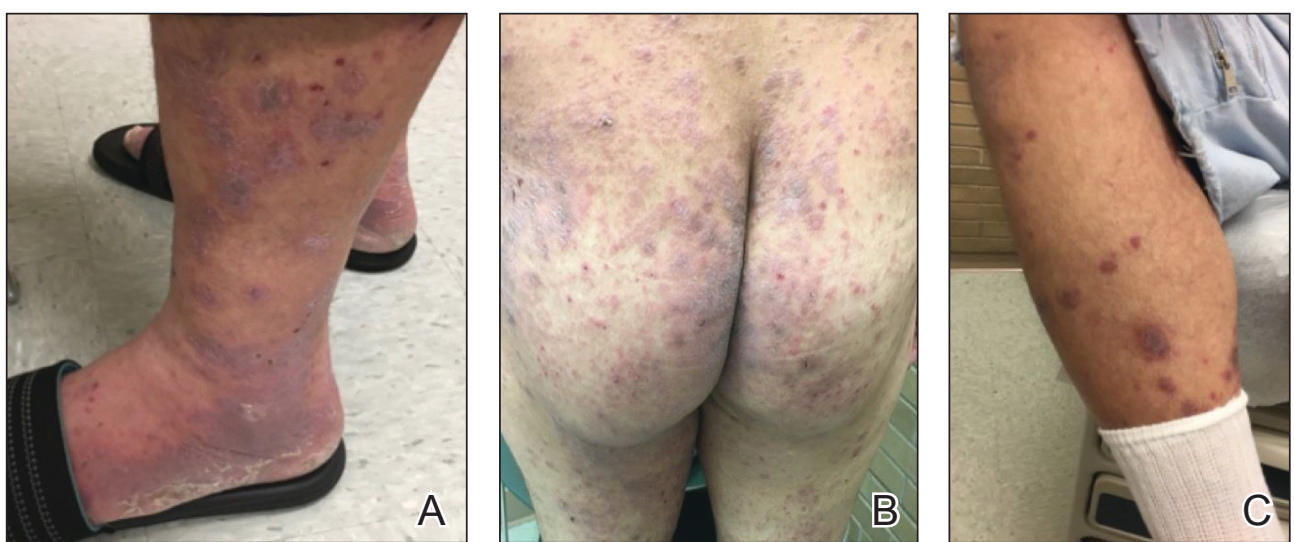

FIGURE 1. A-C, Lesion burden of lichen planus before therapy on the legs and buttocks.
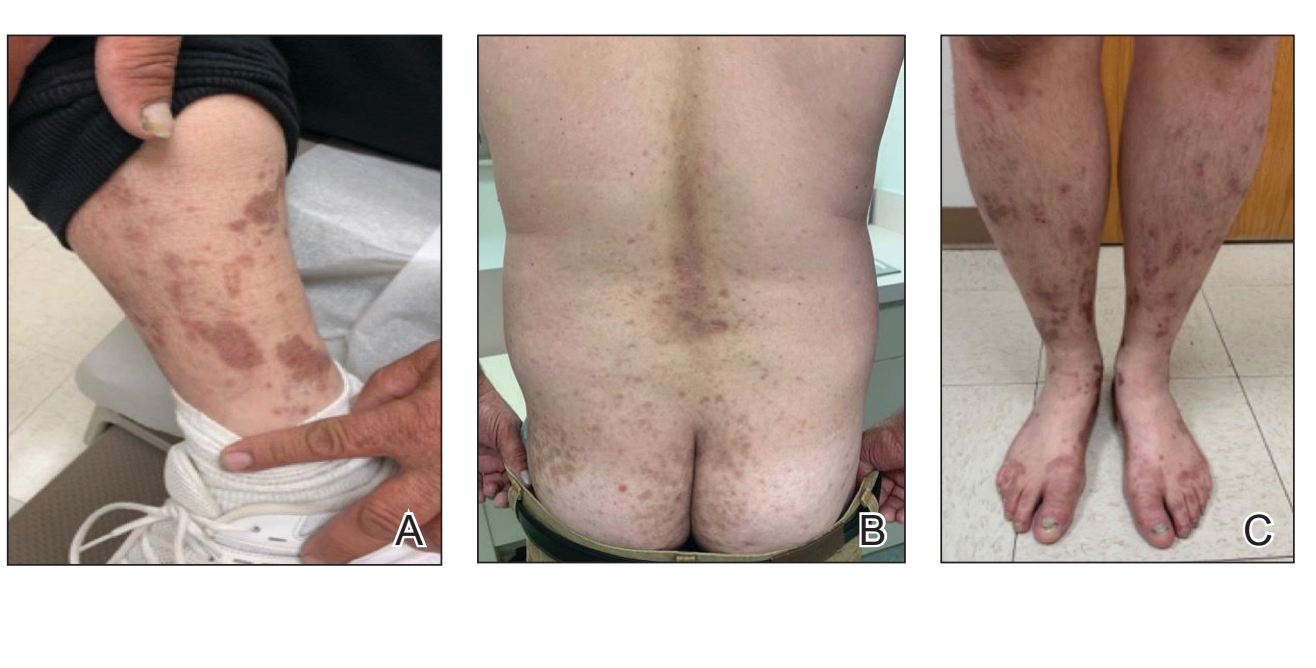

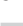

FIGURE 2. A-C, Following dupilumab therapy, the patient experienced decreased lesion burden with residual postinflammatory hyperpigmentation.

Due to the chronic, severe, and recalcitrant nature of his condition, as well as the lack of US Food and Drug Administration-approved treatments, the patient agreed to begin off-label treatment with dupilumab. Upon documentation, the patient's primary diagnosis was listed as LP, clearly stating all commonly accepted treatments were attempted, except off-label therapy, and failed, and the plan was to treat him with dupilumab as if he had a severe form of atopic dermatitis. Dupilumab was approved with this documentation with a minimal co-pay, as the patient was on Medicaid. At 3-month follow-up (after 4 administrations of the medication), the patient showed remarkable improvement in appearance, and his numeric rating scale itch intensity score improved to $1 / 10$.

Lichen planus is an immune-mediated, inflammatory condition that can affect the skin, hair, nails, and oral cavity. Although its etiology is not fully understood, research supports a primarily $\mathrm{T}_{\mathrm{H}} 1$ immunologic reaction. ${ }^{10}$ These $\mathrm{T}$ cells promote cytotoxic CD8 T-cell differentiation and migration, leading to subsequent destruction of epidermal basal keratinocytes. An important cytokine in this pathway-tumor necrosis factor $\alpha$-stimulates a series of proinflammatory factors, including IL- $1 \alpha$, IL-8, and IL-6. IL-6 is of particular interest, as its elevation has been identified in the serum of patients with LP, with levels correlating to disease severity. ${ }^{11}$ This increase is thought to be multifactorial and a reliable predictor of disease activity. ${ }^{12,13}$ In addition to its proinflammatory role, IL-6 promotes the activity of IL-4, an essential cytokine in $\mathrm{T}_{\mathrm{H}} 2$ T-cell differentiation.

The $\mathrm{T}_{\mathrm{H}} 2$ pathway, enhanced by IL-6, increases the activity of downstream cytokines IL-4, IL-5, and IL-13. This pathway promotes IgE class switching and eosinophil maturation, pivotal factors in the development of atopic conditions such as allergic rhinitis, asthma, and atopic dermatitis. The role of IL-4 and $\mathrm{T}_{\mathrm{H}} 2$ cells in the pathogenesis of LP remains poorly understood. ${ }^{14}$ In prior basic laboratory studies, utilizing tissue sampling, RNA extraction, and real-time polymerase chain reaction assays, Yamauchi et $\mathrm{al}^{15}$ proposed that $\mathrm{T}_{\mathrm{H}} 2$-related chemokines played a pathogenic role in oral LP. Additional reports propose the pathogenic involvement of $\mathrm{T}_{\mathrm{H}} 17$, $\mathrm{T}_{\mathrm{H}} 0$, and $\mathrm{T}_{\mathrm{H}} 2 \mathrm{~T}$ cells. ${ }^{16}$ These findings suggest that elevated IL-6 in those with LP may stimulate an increase in IL-4 and subsequent $\mathrm{T}_{\mathrm{H}} 2$ response. Dupilumab, a monoclonal antibody that targets IL-4R $\alpha$ found on T cells, inhibits both IL-4 and IL-13 signaling, decreasing subsequent effector cell function. ${ }^{17,18}$ Several case reports have described dupilumab successfully treating 
various additional dermatoses, including prurigo nodularis, chronic pruritus, and bullous pemphigoid..$^{5-9}$ Our case demonstrates an example of LP responsive to dupilumab. Our findings suggest that dupilumab interacts with the pathogenic cascade of LP, potentially implicating the role of $\mathrm{T}_{\mathrm{H}} 2$ in the pathophysiology of LP.

Treatment-refractory LP remains difficult to manage for both the patient and provider. Treatment regimens remain limited to small uncontrolled studies and case reports. Although primarily considered a $\mathrm{T}_{\mathrm{H}} 1$-mediated disease, the interplay of various alternative signaling pathways has been suggested. Our case of dupilumabresponsive LP suggests an underlying pathologic role of $\mathrm{T}_{\mathrm{H}} 2$-mediated activity. Dupilumab shows promise as an effective therapy for refractory LP, as evidenced by our patient's remarkable response. Larger studies are warranted regarding the role of $\mathrm{T}_{\mathrm{H}} 2$-mediated inflammation and the use of dupilumab in LP.

\section{REFERENCES}

1. Cleach LL, Chosidow O. Clinical practice. lichen planus. N Engl J Med. 2012;266:723-732.

2. Lehman, JS, Tollefson MM, Gibson LE. Lichen planus. Int J Dermatol. 2009;48:682-694

3. Frieling U, Bonsmann G, Schwarz T, et al. Treatment of severe lichen planus with mycophenolate mofetil. J Am Acad Dermatol. 2003;49:1063-1066.

4. Cribier B, Frances C, Chosidow O. Treatment of lichen planus. an evidence-based medicine analysis of efficacy. Arch Dermatol. 1998;134:1521-1530.

5. Calugareanu A, Jachiet C, Lepelletier C, et al. Dramatic improvement of generalized prurigo nodularis with dupilumab. J Eur Acad Dermatol Venereol. 2019;33:E303-E304.
6. Kaye A, Gordon SC, Deverapalli SC, et al. Dupilumab for the treatment of recalcitrant bullous pemphigoid. JAMA Dermatol. 2018;154:1225-1226.

7. Mollanazar NK, Qiu CC, Aldrich JL, et al. Use of dupilumab in HIV-positive patients: report of four cases. Br J Dermatol. 2019; 181:1311-1312.

8. Zhai LL, Savage KT, Qiu CC, et al. Chronic pruritus responding to dupilumab—a case series. Medicines (Basel). 2019;6:72.

9. Mollanazar NK, Elgash M, Weaver L, et al. Reduced itch associated with dupilumab treatment in 4 patients with prurigo nodularis. JAMA Dermatol. 2019;155:121-122.

10. Lodi G, Scully C, Carrozzo M, et al. Current controversies in oral lichen planus: report of an international consensus meeting. part 1. viral infections and etiopathogenesis. Oral Surg Oral Med Oral Pathol Oral Radiol Endod. 2005;100:40-51

11. Yin $\mathrm{M}, \mathrm{Li} \mathrm{G}$, Song $\mathrm{H}$, et al. Identifying the association between interleukin-6 and lichen planus: a meta-analysis. Biomed Rep. 2017;6:571-575.

12. Sun A, Chia JS, Chang YF, et al. Serum interleukin-6 level is a useful marker in evaluating therapeutic effects of levamisole and Chinese medicinal herbs on patients with oral lichen planus. J Oral Pathol Med. 2002;31:196-203.

13. Rhodus NL, Cheng B, Bowles W, et al. Proinflammatory cytokine levels in saliva before and after treatment of (erosive) oral lichen planus with dexamethasone. Oral Dis. 2006;12:112-116.

14. Carrozzo M. Understanding the pathobiology of oral lichen planus. Curr Oral Health Rep. 2014;1:173-179.

15. Yamauchi M, Moriyama M, Hayashida JN, et al. Myeloid dendritic cells stimulated by thymic stromal lymphopoietin promote Th2 immune responses and the pathogenesis of oral lichen planus. Plos One. 2017:12:e173017.

16. Piccinni M-P, Lombardell L, Logidice F, et al. Potential pathogenetic role of Th17, Th0, and Th2 cells in erosive and reticular oral lichen planus. Oral Dis. 2013:20:212-218.

17. Kidd P. Th1/Th2 balance: the hypothesis, its limitations, and implications for health and disease. Altern Med Rev. 2003;8:223-246.

18. Noda S, Kruefer JG, Guttum-Yassky E. The translational revolution and use of biologics in patients with inflammatory skin diseases. J Allergy Clin Immunol. 2015;135:324-336. 